\title{
MOBILISATIONS FONCIÈRES EN TUNISIE : RÉVÉLATEUR DES PARADOXES DE L'APRÈS « RÉVOLUTION »
}

\author{
Alia Gana et Marouen Taleb \\ Article paru dans L'Harmattan | «Confluences Méditerranée », 2019/1 N 108 | pages 31 à 46, ISSN \\ 1148-2664, ISBN 9782343173504
}

Alia Gana est directrice de recherche au CNRS, Principal Investigator, ERC Advanced Grant TARICA.

Marouen Taleb est post-doctorant ERC TARICA.

\section{Résumé}

Au lendemain de la révolte populaire de 2011, les campagnes tunisiennes ont connu d'importantes mobilisations autour de l'accès à la terre qui ont souvent pris des formes violentes. Elles témoignent de l'exacerbation des conflits entre acteurs du monde agricole aux intérêts divergents et de l'ouverture de nouvelles structures d'opportunités pour des groupes jusque-là marginalisés par l'action publique et syndicale. S'intéressant plus particulièrement aux mobilisations autour des terres domaniales agricoles, cet article en explore les enjeux pour les différents acteurs impliqués (paysans sans terre, ouvriers agricoles). Les stratégies et formes d'action déployées par ces derniers et les confrontations auxquelles elles donnent lieu illustrent les reconfigurations socio-politiques à l'œuvre depuis 2011 et révèlent les paradoxes des processus de changement post-révolution en Tunisie.

\section{Abstract}

In the aftermath of the 2011 popular revolt, the Tunisian countryside witnessed major mobilizations around access to land, which often took violent forms. They demonstrate the exacerbation of conflicts between actors of the agricultural world with divergent interests. They also reveal new opportunities for groups hitherto marginalized by public policies and trade union action. With a focus on mobilizations around agricultural land, this article explores the issues at stake for various actors (landless farmers, agricultural workers, etc.). The strategies and forms of action deployed by the latter and the confrontations they generate illustrate the socio-political reconfigurations at work since 2011 and highlight the paradoxes of the post-revolution processes in Tunisia.

\section{Introduction}

Les analyses les plus courantes de la « révolution» tunisienne ont le plus souvent négligé ses racines rurales et agricoles ${ }^{1}$. Pourtant, bien avant le déclenchement de la révolte populaire les conflits en milieu rural étaient largement perceptibles, mais rarement relayés par les médias. Générés par la privatisation des coopératives, le désengagement de l'État de la gestion de l'eau agricole, les menaces d'expropriation pesant sur les agriculteurs endettés, et la hausse des coûts de production, ces conflits donnaient lieu à des mouvements de protestation fortement réprimés par le régime.

Au lendemain de la fuite de Ben Ali, les mobilisations rurales allaient gagner en intensité, engendrant des formes d'action inédites : occupation de terres et de locaux de l'administration, grèves d'ouvriers agricoles, éviction de représentants du syndicat agricole UTAP (Union Tunisienne de l'Agriculture et

\footnotetext{
${ }^{1}$ Elloumi Mohamed, «Les terres domaniales en Tunisie », Études rurales, n 192, 2013, pp. 43-60 ; Gana Alia, « The rural and agricultural roots of the tunisian revolution : when food security matters », International Journal of sociology of Agriculture, ${ }^{\circ}$ 2, volume 19, 2012, pp. 201-213; Gana Alia, 2013 «Aux origines rurales et agricoles de la Révolution tunisienne», Maghreb - Machrek, n²15, volume 1, 2013, pp. 57-80 ; Gana Alia, «Protestations et action collective en milieu agricole et rural. Enjeux et paradoxe du processus de changement politique en Tunisie », Allal Amin, \& Geisser Vincent (dir), Tunisie. Une démocratisation au-dessus de tout soupçon ? 2018, pp. 5772, Paris, CNRS Editions.
} 
de la Pêche $)^{2}$ et d'associations d'usagers de l'eau. Si les revendications exprimées revêtent alors une grande diversité, celles relatives à l'accès à la terre, en particulier aux propriétés de l'État, y occupent une large place. Puissant révélateur des enjeux que représente le contrôle de la gestion des terres domaniales agricoles (TDA), les mobilisations autour du foncier et les confrontations qu'elles suscitent illustrent de manière plus générale la reconfiguration des rapports de force entre acteurs sociaux. Les dynamiques que génèrent les mobilisations foncières mettent aussi en lumière les paradoxes de la transition politique en Tunisie, caractérisée à la fois par l'ouverture de nouveaux espaces d'action pour divers groupes sociaux et les velléités de reprise en main et de mise sous tutelle des organisations syndicales par les autorités politiques.

Dans cet article nous proposons d'explorer les mobilisations autour des TDA comme un espace de conflit, de confrontation et de reconfiguration des rapports de force entre acteurs aux intérêts divergents.

Après avoir rappelé quelques-unes des dynamiques qui ont contribué à la montée des conflits autour des TDA, nous rendons compte des mobilisations auxquelles ces conflits ont donné lieu après 2011 et analysons les logiques sociales qui guident les stratégies des différents acteurs qui y sont impliqués.

\section{Conflits fonciers et mobilisations autour des terres domaniales}

\section{La privatisation de la gestion du domaine agricole de l'État}

Rappelons tout d'abord que la nationalisation des fermes coloniales en 1964 et la récupération de 800000 hectares de terres situés en majorité dans les régions les plus fertiles du nord du pays ont fait de l'État tunisien le plus gros propriétaire foncier. Durant la période allant de 1964 à 1969, cette réserve foncière a ainsi permis la constitution d'unités coopératives de production agricole à partir du regroupement de petits exploitants de moins de 40 ha, autour d'anciennes fermes coloniales. Conçue comme un moyen de favoriser la modernisation technique de l'agriculture, l'expérience coopérative a cependant suscité dès le départ l'hostilité des petits agriculteurs puisqu'elle a consisté, de fait, à les priver de leurs moyens d'existence (terre et cheptel). En 1969, l'annonce de la généralisation de la forme coopérative à l'ensemble de l'agriculture a provoqué une réaction farouche des grands agriculteurs, dès le départ opposés à une réforme menaçant la propriété privée des terres et dont la forte mobilisation a fini par sceller le sort de l'expérience. Son arrêt permet aux petits agriculteurs, fortement affaiblis, de récupérer leurs terres tandis que sur les terres appartenant à l'État, les coopératives restructurées sont alors maintenues. Elles continuent à coexister avec les fermes pilotes et les agro-combinats, exploitations gérées directement par l'Office des terres domaniales (OTD).

Cet état de fait va évoluer avec le tournant libéral du milieu des années 1980, sur fond d'ajustement structurel. En effet, un processus de privatisation de la gestion des TDA est alors lancé. La gestion d'une large partie des coopératives est ainsi transférée principalement à deux groupes de bénéficiaires : les techniciens et ingénieurs agronomes et les sociétés de mise en valeur et de développement agricole (SMVDA) qui bénéficieront d'importants investissements grâce à la mise en place de banques spécialisées. Mais ce transfert de la gestion des terres de l'État à des promoteurs privés issus du monde urbain, est perçu par les locaux comme un processus de colonisation par des élites autochtones nourrissant le ressentiment des anciens « coopérateurs » et des petits agriculteurs avoisinants. Plus encore, il est à l'origine de nombreuses tensions entre les ouvriers agricoles et les gestionnaires des sociétés de mise en valeur ${ }^{3}$.

\footnotetext{
${ }^{2}$ Ce syndicat agricole unique jusqu'en 2011 avait été créé en 1955 sous le nom d'Union nationale des agriculteurs tunisiens (UNAT).

${ }^{3}$ Bien que le cahier des charges imposait aux nouveaux gestionnaires de maintenir l'emploi des ouvriers agricoles des anciennes coopératives, une bonne part de ces derniers ont perdu leur travail, ainsi que la possibilité qu'ils avaient de maintenir un petit troupeau et de prélever différentes ressources sur la coopérative (Gana, 2012, op. cité).
} 


\section{L'occupation des terres ou la réactivation d'une mémoire collective de la spoliation}

Dans ces conditions, il n'y a rien d'étonnant à ce que le soulèvement populaire de 2010-2011 ait réactivé le sentiment d'injustice engendré par l'exclusion des petits paysans et ouvriers agricoles du processus de privatisation des coopératives. Ainsi, au lendemain du 14 janvier 2011, des fermes gérées par des SMVDA ou par des ingénieurs agricoles (lots techniciens) ont été occupées ou ont été la cible de pillages (vol de bétail) et d'importantes destructions (équipements, dégradation des récoltes). Ces actions ont touché plus d'une centaine de fermes, situées dans presque toutes les régions, et plusieurs dizaines de milliers d'hectares. Selon les responsables des domaines de l'État, les actes de vandalisme sur les SMVDA et les lots techniciens ont souvent été le fait des jeunes des bourgs ruraux limitrophes, pour qui les engagements pris par les attributaires d'assurer un nombre d'emploi minimum, en recrutant dans le voisinage de la ferme, et d'investir durablement dans la terre n'ont pas été tenus ${ }^{4}$.

Les occupations de terre ont également touché des SMVDA dont les gestionnaires avaient été déchus de leurs droits d'exploitation au lendemain de la révolution pour non-respect du cahier des charges.

Bien que réintégrées à l'OTD, les fermes concernées ont été occupées par des riverains (petits agriculteurs et sans terre) justifiant leurs actions par l'état d'abandon des terres et la nécessité de les revivifier ${ }^{5}$.

Depuis 2011, les mobilisations autour du foncier vont même jusqu'à remettre en cause la propriété étatique des terres agricoles. En effet, dans plusieurs régions, des paysans revendiquent aujourd'hui de pouvoir récupérer les terres «de leurs ancêtres », confisquées tout d'abord par les colons français et ensuite nationalisées par l'État après l'indépendance. Des familles ont de la sorte entrepris des démarches pour la reconnaissance de leurs droits sur des terres de l'État en s'appuyant sur la présentation de titres anciens ${ }^{6}$.

\section{Les mobilisations d'ouvriers agricoles et la dialectique syndicale}

Au lendemain du 14 janvier 2011, plusieurs mouvements de grève et des sit-in sur les lieux de travail ont été aussi organisés par des ouvriers agricoles de SMVDA pour demander une amélioration de leurs conditions de travail et de rémunération. Bien que parfois soutenues par des sections régionales de l'UGTT, les mobilisations d'ouvriers agricoles ont surtout eu un caractère spontané et souvent revêtu des formes violentes (occupations, menaces de destruction de l'outil de production).

Une des revendications formulées par les ouvriers des SMVDA portait sur la création de groupements de travailleurs agricoles, au sein desquels ces derniers pourraient s'organiser et faire entendre leur voix ${ }^{7}$. Ce projet d'organisation autonome a été mal accueilli par l'UGTT qui, dès lors, s'est davantage investie dans le soutien aux luttes des ouvriers agricoles. Ce soutien s'est notamment manifesté dans le gouvernorat de Siliana au nord ouest de la Tunisie lors des conflits opposant des ouvriers agricoles aux gestionnaires de la SMVDA Lakhmès SODAL, ainsi que lors de mobilisations des ouvriers de la ferme d'El Krib, gérée par l'OTD.

Dans le cas de la ferme Lakhmès gérée par la SODAL, le conflit était lié aux graves difficultés financières de l'entreprise qui l'ont placée devant l'incapacité de payer les salaires de ses 100 employés. Les mobilisations que cette situation n'a pas manqué de déclencher ont donc été fortement soutenues par l'UGTT, la centrale syndicale pesant de tout son poids dans les négociations avec l'administration

\footnotetext{
${ }^{4}$ Entretien avec un responsable du ministère du Domaine de l'État et des affaires foncières.

${ }^{5}$ Entretien avec un responsable du ministère des Domaines de l'État et des affaires foncières.

${ }^{6}$ African Manager, «Tunisie : Terres agricoles et investisseurs, cherchent protection désespérément !», 2011, <http://farmlandgrab.org/post/print/18854>;

${ }^{7}$ Gana, 2013, op.cité
} 
régionale et les ministères concernés. Les pressions qu'elle a exercées ont finalement abouti à la déchéance des droits d'exploitation de la SODAL et à la réintégration de la ferme au domaine géré par l'OTD $^{8}$. Si cette décision a consacré une première victoire de l'UGTT et des ouvriers agricoles dans leur bataille contre les privatisations, elle a représenté un revers pour le principal syndicat agricole (UTAP), qui, réintégré dans le giron du pouvoir après la vague de contestation dont elle a fait l'objet en 2011, s'est positionné plutôt du côté des gestionnaires des SMVDA.

C'est cette position que l'UTAP a également défendue lors du conflit autour de l'agro-combinat Mohsen Liman à El Krib (gouvernorat de Siliana). En septembre 2018, une série de photographies et de vidéos montrant une quarantaine de vaches laitières mortes, appartenant supposément à l'agro-combinat géré par l'OTD, sont apparues sur les réseaux sociaux et relayées par les médias nationaux ${ }^{9}$. L'UTAP est alors la première à s'emparer de l'affaire en dénonçant la mauvaise gestion de la ferme, confrontée depuis plusieurs années à des difficultés financières et à la baisse de sa production animale et végétale ${ }^{10}$. Pour ce syndicat, il fallait se saisir de l'occasion pour réaffirmer sa position en faveur de la privatisation des agro-combinats, et cela en mettant en avant l'incapacité de l'État à gérer directement l'activité agricole.

Parallèlement au démenti apporté par l'administration concernant les pertes enregistrées dans le cheptel bovin, une mobilisation des gestionnaires et des ouvriers agricoles de l'agro-combinat est alors organisée avec l'appui de l'UGTT pour dénoncer les informations relayées par l'UTAP. Il s'agit de répondre à ce qui est considéré comme une attaque en règle contre le secteur public et par conséquent de "rétablir l'image de l'agro-combinat ternie par la campagne orchestrée par l'UTAP et par les médias ${ }^{11}$ », accusés de servir de façade à des acteurs privés voulant s'approprier les fermes d'État.

Si les «affaires » de la SMDVA «Lakhmes » et de l'agro-combinat «Mohsen Liman » ne permettent pas d'anticiper sur le sort que les pouvoirs publics réserveront à l'avenir aux fermes domaniales, elles témoignent de l'exacerbation des conflits entre acteurs agricoles aux intérêts divergents, en particulier, ici, entre partisans de la privatisation et défenseurs du domaine public agricole.

\section{Les gestionnaires des SMVDA et des lots techniciens : préserver les droits acquis}

Face à la remise en cause du modèle de gestion privée des terres domaniales agricoles et en réaction aux attaques et aux destructions subies au lendemain de la « révolution», les gestionnaires des SMVDA et des lots techniciens se sont organisés pour défendre leurs intérêts. C'est au sein de l'UTAP et plus particulièrement de la Fédération nationale des sociétés de développement et de mise en valeur agricole (FN-SMVDA) qu'ils se sont mobilisés pour faire valoir leurs demandes d'indemnisations pour les pertes subies suite aux occupations sauvages, pour réclamer la protection de leurs droits en tant que locataires légaux, et l'adoption de mesures leur permettant de retourner sur leurs fermes et de reprendre leurs activités. À plusieurs reprises, la fédération des SMVDA a dénoncé la lenteur de la mise à exécution des mesures obtenues pour évacuer les «squatteurs » de leurs fermes, appelant les autorités publiques à clarifier leur position sur les actes de vandalisme et allant jusqu'à les accuser de cautionner implicitement les projets politiques appelant à la restructuration des terres domaniales.

\footnotetext{
${ }^{8}$ Communiqué du ministère des Domaines de l’État en date du 7 septembre 2018, Mosaïquefm.net, « Siliana: La gestion de la ferme 'Lakhmès' confiée à l'OTD », Tunis, 7 septembre 2018.

${ }^{9}$ Chaînes radio Mosaïque Fm et Shems Fm.

10 Déclaration du chef de la section locale de l'UTAP à El Krib en date du 18 septembre 2018, https://www.mosaiquefm.net.

${ }^{11}$ Avis de grève de l'UGTT Siliana en date du 26 septembre 2018.
} 
Pendant plusieurs mois, leurs requêtes ont été peu entendues par l'Administration, celle-ci hésitant à accéder à des demandes peu populaires et émanant de surcroît d'un groupe organisé au sein d'une fédération de l'UTAP, considérée comme un symbole de l'ancien régime ${ }^{12}$.

Cette attitude a contribué à aggraver la situation financière des fermes domaniales gérées par des entreprises privées suite à une accumulation des dettes contractées auprès de l'État pour non-paiement des loyers. Très peu critique vis-à-vis des pouvoirs publics entre 2011 et 2014 durant le gouvernent de la Trö̈ka ${ }^{13}$, l'UTAP, contrôlée désormais par les réseaux du parti islamiste Ennahdha, s'est dès lors mobilisée à de nombreuses reprises à partir de 2015 auprès de l'Assemblée des Représentants du Peuple $\left(\mathrm{ARP}^{14}\right)$ et du Ministère des domaines de l'État pour réclamer un rééchelonnement des dettes des SMVDA. L'engagement de l'UTAP aux côtés des SMVDA s'explique par le fait que les nouvelles attributions de TDA à des promoteurs privés tendent de nouveau à privilégier des promoteurs proches $\mathrm{du}$ pouvoir politique, notamment du parti islamiste ${ }^{15}$. C'est dans ce contexte que la loi ${ }^{\circ}$ 08-2016 relative au rééchelonnement des dettes des locataires d'immeubles domaniaux agricoles a été promulguée le 22 février 2016.

\section{Les pouvoirs publics face aux mobilisations foncières : de l'attentisme à la reprise en main}

La réaction des pouvoirs publics face aux occupations de terres et leur position quant au sort à réserver aux gestionnaires privés des terres domaniales a été caractérisée par une évolution notable depuis 2011. Elle reflète les reconfigurations politiques qui marquent le processus de « démocratisation » en Tunisie depuis 2011.

\section{Solder les pratiques de l'ancien régime?}

Les premières mesures prises par le gouvernement de la Troïka (Ennahda, CPR et Ettakatol), visant surtout à réduire les tensions autour des TDA, ont consisté dans la récupération des SMVDA gérées par des proches de l'ancien régime, avec reprise des actions par l'État. Au cours de l'année 2012, les commissions régionales de confiscation et de récupération des TDA ont procédé à la déchéance de droits pour 33 SMVDA (22377 hectares) dont la gestion a été confiée provisoirement à l'OTD. Le rythme des confiscations par déchéance du droit d'exploitation s'est accéléré ensuite pour atteindre en 2016 le nombre de 178 SMVDA, soit 73446 hectares $^{16}$.

Durant la période transitoire et celle qui a suivi l'accession au pouvoir de la Troïka, les confiscations touchant d'anciens gestionnaires de SMVDA ont été présentées comme des mesures visant à rompre avec le népotisme et les symboles de l'ancien régime. Jouant sur les effets d'annonce que constituent les mises sous séquestre, la nouvelle administration avait, en fait, comme objectif de placer les TDA progressivement sous son contrôle afin d'accroître sa marge de manœuvre pour appliquer ses orientations de politique agricole. En effet, loin de remettre en cause les privatisations, les nouvelles autorités ont très tôt réaffirmé les options en faveur de la libéralisation du secteur agricole ${ }^{17}$ et ont surtout

\footnotetext{
12 Gana 2013, op.cité

${ }^{13}$ Gouvernement issu des élections d'octobre 2011 formé par une alliance entre le parti islamistes Ennahdha, majoritaire, et deux partis de centre-gauche (octobre 2011-janvier 2014).

14 Parlement élu en 2014.

${ }^{15}$ Gana, 2018, op.cité

${ }^{16}$ DGIA - Ministère des Domaines de l'État et des Affaires Foncières.

${ }^{17}$ Dès le départ, le gouvernement de la Troïka affirme sa volonté d'encourager les investissements étrangers dans l'agriculture, en particulier ceux provenant des pays du Golfe, investissements présentés comme le moyen d'intensifier la production agricole et de créer des emplois pour la population rurale. Durant cette période plusieurs accords de financement de projets agricoles sur les terres de l'État ont été conclus avec les pays du Golfe.
} 
cherché, comme on le verra plus loin, à faire bénéficier les proches et les alliés du parti islamiste des nouvelles attributions de terres, dans le but de renforcer leurs assises économiques. Cette logique explique la lenteur des procédures de réhabilitation dans leurs droits des gestionnaires ayant fait l'objet d'attaques et d'occupations et les freins mis en place pour empêcher leur retour sur les exploitations ${ }^{18}$.

\section{Le lent processus de récupération des terres domaniales}

Dans le souci d'accréditer auprès des électeurs sa fidélité à un projet politique de rupture avec l'ancien régime et de ne pas s'aliéner le soutien des groupes se réclamant du courant " révolutionnaire », le nouveau pouvoir politique issu des élections de 2011 adopte une attitude attentiste et dissuade l'administration de recourir à la force publique pour faire appliquer les décisions d'expulsion émises par la justice à l'encontre des occupants de terres. Des entretiens avec des responsables du Ministère des Domaines de l'État révèlent que, durant les premières années qui ont suivi la révolution, les directions régionales du ministère ont été souvent soumises à des pressions exercées par les autorités politiques qui intercédaient «pour raison morale » en faveur d'exploitants « illégaux » en vue de geler ou surseoir à l'exécution d'arrêtés d'expulsion.

En fait, c'est seulement après 2014, avec l'accession au pouvoir de la nouvelle coalition gouvernementale $^{19}$, que le processus de récupération des terres squattées connaît une accélération. Dans la logique de la campagne qu'il a menée lors des élections de 2014, le parti vainqueur (Nida Tounes) a réaffirmé son projet de mettre en œuvre « une stratégie nationale de restauration de l'autorité de l'État ». Par conséquent, c'est sous le slogan de la « lutte contre l'impunité » face à la "spoliation des biens de l'État » que les pouvoirs publics vont désormais inscrire le processus de récupération des terres occupées. En 2016, le Ministère des domaines de l'État a procédé à un recensement détaillé des terres occupées et évalué leurs superficies à 20330 hectares, la priorité a été donnée à la récupération de 21 SMVDA, implantées dans les zones les plus fertiles et dont la location assurait à l'État des revenus conséquents. L'application des arrêtés d'expulsion des terres de l'État émis par les gouverneurs a souvent été marquée par des affrontements entre les familles d'agriculteurs et la force publique. Toutefois, de nombreux occupants, souvent des agriculteurs riverains qui se savaient en infraction, ont restitué de leur plein gré les terres qu'ils exploitaient depuis la révolution ${ }^{20}$.

Si dans la période récente, l'action du Ministère des domaines de l'État a permis de remettre sous le contrôle de l'administration (OTD) l'essentiel des terres ayant fait l'objet d'occupations sauvages, la réintégration d'une partie d'entre elles dans le secteur public n'a pas suscité l'adhésion de toute la classe politique. Le syndicat agricole UTAP a eu une réaction plutôt hostile tandis que de fortes résistances se sont exprimées de la part de certaines parties au sein de la coalition au pouvoir. En effet la déchéance des droits contractuels de bon nombre d'anciens gestionnaires a attisé les convoitises de nouveaux acteurs politiques et hommes d'affaires proches des deux grands partis de la coalition gouvernementale (Ennahda et Nida Tounes).

\section{L'attribution des terres domaniales : quand prévaut la logique clientéliste}

Dès l'arrivée au pouvoir de la Troïka en 2011, l'action des nouveaux responsables du ministère des Domaines de l'État a consisté à faciliter la résiliation des contrats de location détenus par les gestionnaires de SMVDA proches de l'ancien régime ou supposés hostiles à la nouvelle coalition gouvernementale. En effet, il ressort de nos entretiens que les services en charge du contentieux au sein

\footnotetext{
${ }^{18}$ Avant toute intervention, l'administration exigeait d'eux le paiement préalable des arriérés de location, alors que leurs parcelles n'étaient plus accessibles et encore moins mises en culture; Office des Terres Domaniales, Rapport d'activité annuel pour la campagne agricole 2015/2016, Tunis, OTD, 2017, p.122.

${ }^{19}$ La nouvelle coalition formée à l'issue des législatives de 2014 associe le parti vainqueur Nida Tounes au parti islamiste Ennahdha.

${ }^{20}$ Communiqués du Ministère des Domaines de l’État.
} 
du Ministère des domaines de l'État ont cherché à exploiter les défaillances des gestionnaires en matière de respect des cahiers des charges, surtout dans le but de libérer les terres concernées et de les remettre en location. Dès septembre 2013, un appel d'offre pour la location de 45 SMVDA récupérées a été publié par l'Agence de Promotion des Investissements Agricoles (APIA). Cet appel d'offre a très vite suscité des contestations en raison des délais très courts imposés aux investisseurs pour présenter leurs dossiers, et de l'obligation qu'il leur était faite de les soumettre en langue arabe. Nos interlocuteurs soulignent que ce changement brusque dans les règles de soumission des dossiers a empêché de nombreux investisseurs de participer à l'appel d'offre, favorisant à l'inverse, « les candidats appartenant aux réseaux de la Troïka qui avaient, eux, une connaissance préalable des conditions et des délais pour la présentation des offres $»^{21}$. Selon des cadres de l'administration publique, « les SMVDA constituaient pour le parti islamiste Ennahdha une opportunité d'investissement pour les capitaux accumulés par la diaspora 'nahdhaoui' et un moyen de renflouer les caisses du parti $»^{22}$.

Depuis 2011, et à travers deux appels d'offres ${ }^{23}$, 43 SMVDA couvrant une superficie de 32154 hectares et localisées dans leur grande majorité dans les régions fertiles du nord de la Tunisie ont été attribuées à de nouveaux gestionnaires. Parmi ces derniers, on trouve des membres de la famille et des proches du parti islamiste comme cela a été suggéré plus haut, mais aussi des proches et membres du parti Nida Tounes, des hommes d'affaires et des dirigeants de clubs de football ayant fait leur entrée en politique, des hauts cadres de l'administration publique et des hauts commis de l'État.

Contrairement aux SMVDA dont la redistribution a reçu très tôt l'attention des pouvoirs publics, les attributions de lots techniciens et ingénieurs agricoles n'ont été concrétisées qu'en mars 2018 et ont concerné 36 lots techniciens couvrant une superficie de 1092 hectares. Quant aux attributions au profit de jeunes agriculteurs, interrompues entre 2011 et 2017, elles n'ont porté depuis la publication de la circulaire du 9 février $2017^{24}$ que sur 42 lots couvrant au total 406,55 hectares ${ }^{25}$. Ces attributions continuent d'être guidées par des objectifs à caractère social ${ }^{26}$, voire par une logique clientéliste, visant à atténuer les tensions sociales autour de l'accès à la terre et de l'emploi ${ }^{27}$.

\section{Quelles perspectives en matière de gestion du domaine agricole de l'État? Entre projets alternatifs et modèles néolibéraux}

Ainsi, malgré les fortes mobilisations autour du foncier, l'ascension au pouvoir de nouvelles élites politiques et les coalitions gouvernementales formées à l'issue des élections de 2011 et 2014 continuent d'opérer en faveur de la privatisation des TDA au profit de grands investisseurs ou de proches du régime. Rien d'étonnant dès lors à ce que l'action gouvernementale soit soumise à de fortes contestations de la part d'acteurs revendiquant un meilleur accès à la terre et qui ont pu s'assurer le soutien de la société civile et de partis politiques de l'opposition. Leur mobilisation a permis à des projets innovants de gestion des TDA, appuyés par des associations, de voir le jour. La médiatisation de ces expériences a

\footnotetext{
${ }^{21}$ Entretiens avec des responsables l'APIA et de l'OTD.

${ }^{22}$ Des particuliers, servant de prête-noms, auraient déposé des dossiers pour la location de SMVDA avec une participation majoritaire d'actionnaires membres du parti Ennahda (entretiens).

${ }^{23}$ Liste 34 publiée le 21 janvier 2014 et liste 35 publiée le 15 mars 2017.

${ }^{24}$ Circulaire conjointe des Ministres de l'Agriculture et des Domaines de l'État portant sur l'attribution de lots jeunes agriculteurs, en date du 09 février 2017.

${ }^{25}$ Entretien avec un responsable du bureau de restructuration des terres domaniales.

${ }^{26}$ Gharbi Inès, Elloumi Mohamed, Jamin, Jean.-Yves \& Maayoufi Dorsaf, « L'attribution de terres domaniales irriguées aux jeunes ruraux en Tunisie : création d'emplois durables ou mise en place d'exploitations non viables ? $\gg \quad$ Cahiers $\quad$ Agricultures, $\mathrm{n}^{\circ} \quad 27, \quad$ volume $4, \quad 48$ https://www.cahiersagricultures.fr/articles/cagri/full_html/2018/04/cagri170197/cagri170197.html.

${ }^{27}$ Entretiens avec des responsables du Ministère de l'Agriculture et des services régionaux du Ministère des Domaines de l'État.
} 
contribué à infléchir quelque peu les positions des pouvoirs publics, sans toutefois remettre cause les grandes orientations du développement.

\section{La palmeraie de Jemna : vers l'émergence d'un nouvel modèle de gestion des TDA?}

Si pendant plusieurs années les revendications autour des TDA ont été peu entendues par les pouvoirs publics et ont eu peu d'écho dans les médias, récemment l'affaire de la ferme domaniale Jemna ${ }^{28}$ gérée par une association locale, a suscité des controverses politiques largement médiatisées.

Dès janvier 2011, des habitants de Jemna, soutenus par un «comité local de protection de la révolution », ont occupé une palmeraie relevant du domaine agricole de l'État, dont la gestion sous forme de SMVDA était auparavant assurée par deux promoteurs privés, soupçonnés de corruption et de proximité avec le régime de Ben Ali.

Ayant opté pour une gestion collective, un groupe d'activistes constitué d'enseignants et de militants politiques originaires de la localité de Jemna, ont créé, en mars 2012, une association pour prendre en charge l'exploitation de la ferme. Forte du soutien des habitants et mettant en avant la légitimité historique de leur action pour « récupérer la terre de leurs ancêtres, spoliés par la colonisation et par l'État ", l'association a assuré avec grand succès la gestion de la palmeraie sans être inquiétée par les pouvoirs publics jusqu'en 2016. Malgré les décisions de justice émises en faveur de l'État, l'autorité régionale n'a pas été en mesure de les mettre à exécution, par crainte des troubles qu'aurait générés une intervention des forces de l'ordre et compte tenu de la forte popularité de l'association et de ses réalisations sociales en faveur des habitants de la commune de Jemna.

À la suite de la publication par l'association d'un appel d'offres pour la vente de la production des dattes, en septembre 2016, le conflit entre l'association et le ministère des Domaines de l'État a éclaté au grand jour. Dans un premier temps, ce dernier a rappelé que la ferme appartient à l'État et que « tout contrat conclu concernant cette ferme ou ses produits n'a pas de valeur légale $»^{29}$. Mais, face à la mobilisation des habitants de Jemna et au lancement d'une procédure judiciaire en vue de la reconnaissance de leur droit de propriété sur la ferme, l'administration a été obligée d'engager des négociations. Après avoir proposé la transformation de l'association en SMVDA, solution rejetée par le collectif, la nouvelle formule étudiée pour trouver un cadre réglementaire à la gestion de la palmeraie est la création d'une coopérative de production agricole. En octobre 2017, les concertations engagées ont donné lieu à un accord entre l'association Jemna et le ministère de l'agriculture, qui revient en fait à maintenir le statu quo. Cet accord illustre une évolution importante de la position des pouvoirs publics à l'égard de l'expérience de gestion associative de la ferme Jemna, présentée désormais comme une expérience à préserver et à « organiser au service de l'économie solidaire $»^{30}$.

\section{La ferme Tarfaya : éviter la « contagion »}

Si l'accord conclu avec l'association Jemna a permis une reconnaissance de fait du modèle de gestion collective de la palmeraie, aux yeux de l'administration, et en particulier du ministère des Domaines de l'État, il s'agit de faire en sorte que l'expérience ne fasse pas contagion.

En témoigne la stratégie mise en œuvre par les pouvoirs publics dans le cas de la ferme domaniale Tarfaya. Située également dans le gouvernorat de Kebili à proximité de la frontière algérienne, la ferme Tarfaya est une plantation de 7000 palmiers dattiers couvrant 123 hectares qui fut également occupée par des agriculteurs riverains au lendemain de la "révolution » et exploitée jusqu'en 2016 par un collectif de gestion calqué sur le modèle de l'association Jemna. Comme nous l'avons déjà indiqué, les changements opérés dans l'administration à l'issue des élections 2014 ont consacré une réorientation de

\footnotetext{
${ }^{28}$ Localité du sud tunisien située entre Douz et Kebili.

${ }^{29}$ La Presse, « Secrétariat d'État aux domaines de l'État : les oasis de Jemna sont la propriété de l'État », 2016, http://www.lapresse.tn/component/nationals/?task=article\&id=119424

${ }^{30}$ Web manager center, « Vers la création d'une coopérative à la ferme de STIL à Jemna à Kébili », 2017, https://www.webmanagercenter.com/2017/09/23/410279/vers-la-creation-dune-cooperative-a-la-ferme-de-stil-ajemna-a-kebili/.
} 
l'action du Ministère des domaines de l'État visant à remettre sous le contrôle de l'OTD les terres occupées. C'est ainsi que des négociations furent engagées avec les représentants du comité de gestion de la ferme Tarfaya.

Au départ, les agriculteurs mobilisés au sein de ce comité se sont montrés hostiles à toute solution impliquant la restitution de la ferme à l'État et la renonciation aux revenus conséquents tirés de son exploitation $^{31}$. Par la suite, le collectif de gestion a accepté de restituer la ferme à l'État contre la garantie de bénéficier d'un traitement prioritaire lors de sa mise en concession sous forme de SMVDA. Le placement de la palmeraie sous le contrôle de l'Office de Développement de Regim Maatoug a permis à la grande majorité des agriculteurs de l'ancien collectif de gestion de continuer à travailler sur la plantation, facilitant ainsi sa récupération par l'État. Pour l'administration, la recherche d'une solution négociée aboutissant au retour de la ferme dans le domaine public était guidée par plusieurs objectifs : affirmer le caractère non reproductible du modèle de Jemna, éviter une confrontation avec les habitants d'une zone frontalière stratégique d'un point de vue sécuritaire, s'appuyer sur l'exemple de Tafraya pour lancer une campagne de récupération de l'ensemble des terres domaniales occupées.

\section{La réhabilitation du cadre coopératif pour gérer les terres domaniales agricoles}

La réhabilitation des coopératives comme forme d'exploitation des TDA a été remise à l'ordre du jour après l'affaire de Jemna et la difficulté de trouver un cadre légal pour l'exploitation de la ferme par l'association locale. Pour le ministère de l'Agriculture, confronté aux problèmes de développement dans les régions intérieures, la formule coopérative est envisagée comme un moyen de regrouper des jeunes agriculteurs et des jeunes techniciens agricoles selon une approche d'économie sociale et solidaire.

Ainsi, l'État étudie la possibilité de mettre en location des TDA sous la forme d'Unités coopératives de production agricole. Puisant dans les SMVDA récupérées et celles qui n'ont pas trouvé preneur dans les appels d'offre de l'APIA, le ministère de l'Agriculture a sélectionné 18 fermes pouvant faire l'objet d'une organisation en coopératives, situées dans huit gouvernorats (principalement à Bizerte et à Siliana) et couvrant une superficie totale de 10000 hectares $^{32}$. La réhabilitation du modèle coopératif pour installer de jeunes agriculteurs et techniciens dans le cadre d'exploitations viables témoigne d'une certaine évolution de l'approche des pouvoirs publics quant aux options possibles pour la gestion des fermes récupérées et intégrées provisoirement à l'OTD. La montée des revendications autour des fermes d'État et les mobilisations pour l'accès à l'emploi, mais également la difficulté pour l'OTD de prendre en gestion les terres récupérées expliquent cette évolution.

\section{Le partenariat public-privé : inciter les acteurs privés à investir dans l'agriculture}

Confrontés à la difficulté de prendre directement en charge la gestion du domaine foncier agricole, qui se pose avec plus d'acuité après la réintégration d'anciennes SMVDA à l'OTD, les pouvoirs publics prônent, depuis peu, la formule du partenariat public privé (PPP) pour attirer les capitaux privés tunisiens ou étrangers vers l'agriculture. Sans revenir sur le principe de la non-cessibilité des TDA, cette formule concerne les agro-combinats qui connaissent des difficultés financières. L'État tunisien à travers l'OTD fournirait ainsi le savoir-faire et l'infrastructure existante dans les agro-combinats tandis que le capital privé s'impliquerait par le financement et l'organisation de la commercialisation sur le marché local et international. Le cahier des charges relatif à la formule du PPP a été très critiqué par l'UGTT. Son adoption conjointe par le ministère de l'Agriculture et celui des Domaines de l'État en avril 2017 a donné lieu à une mobilisation de la centrale syndicale ${ }^{33}$ et au lancement d'un mouvement de grève des ouvriers agricoles, dénonçant le risque de voir les investisseurs privés prendre en charge la gestion des

\footnotetext{
${ }^{31}$ Le Ministère du Domaine de l'État a évalué les revenus tirés par le collectif de l'exploitation de la ferme Tarfaya à 400000 DT par an, soit environ 150000 euros au moment de sa restitution.

32 Le Temps, « Mise en location de 45000 hectares de terres domaniales », 2018, http://www.letemps.com.tn/article/107364/mise-en-location-de-45-000-hectaresde-terres-domaniales.

33 Manifeste de la fédération générale de l'Agriculture-UGTT en date du 19 mai 2017) https://www.facebook.com/permalink.php?story_fbid=693582377509856\&id=384379428430154\&_tn__=K-R
} 
ressources humaines et les opérations de restructuration des fermes d'État ${ }^{34}$. Ces mobilisations ont conduit les pouvoirs publics à réviser le cahier des charges relatif à la location des agro-combinats et à limiter les PPP à l'exploitation des TDA récupérées et gérées actuellement par l'OTD à titre provisoire ${ }^{35}$. L'accord passé avec l'UGTT le 19 juin 2017 a permis en outre l'attribution à titre définitif à l'OTD de 7 SMVDA récupérées (6500 hectares) afin de renforcer son équilibre budgétaire et « préserver ainsi les agro-combinats contre toute tentative future de privatisation $»^{36}$.

\section{Conclusion}

Ce tour d'horizon des mobilisations autour des TDA montre la montée en puissance des revendications pour un meilleur accès des ruraux à la terre et à l'emploi. Bien qu'elles n'aient pas suffi à générer une vraie réforme des politiques foncières et agricoles, ces mobilisations ont remis à l'ordre du jour la question foncière, réactivé le débat sur la réforme agraire et suscité une réflexion sur les modèles de développement de l'agriculture. Témoignant de l'exacerbation des conflits entre acteurs du monde agricole aux intérêts divergents et de l'ouverture de nouveaux espaces d'expression pour les groupes jusque-là marginalisés par l'action publique et syndicale, les mobilisations autour des terres de l'État illustrent aussi l'évolution des rapports de force entre partisans de la privatisation et défenseurs du domaine public agricole. Elles mettent également en évidence la fragmentation et les incohérences des politiques publiques oscillant au gré des pressions contradictoires exercées par les acteurs et des changements politiques.

Dans un contexte de profonde reconfiguration de l'espace politique, mais aussi de renforcement de la société civile, les mobilisations foncières ont favorisé l'émergence et la consolidation d'expériences de gestion collective des TDA s'inspirant de l'économie sociale et solidaire. Elles ont ainsi contribué à réhabiliter le modèle coopératif, considéré jusqu'à une date récente comme obsolète, et à infléchir l'action publique dans le sens d'une meilleure prise en compte des demandes sociales autour de l'accès à la terre et à l'emploi. Confrontés depuis 2011aux résistances de la société civile et des ouvriers agricoles du secteur public, les pouvoirs publics n'ont plus les coudées franches pour appliquer sans concertation les formes de gestion agricole inspirées du modèle des partenariats public-privé et préconisées par les bailleurs de fonds internationaux. Si les avancées sur la voie d'une meilleure intégration de la diversité des approches en matière de gestion des TDA sont incontestables, elles ne permettent pas néanmoins d'anticiper sur l'avenir qui leur sera finalement réservé. Celui-ci dépendra de l'évolution du jeu politique, caractérisé aujourd'hui à la fois par une forte instabilité et une certaine ouverture, mais susceptible aussi de se refermer, compte tenu des tentations de restauration autoritaire manifestées par les forces politiques actuellement au pouvoir.

\footnotetext{
${ }^{34} \mathrm{Al}$ Maghreb, [En arabe], « La fédération générale de l'agriculture annonce une grève de trois jours à l'OTD en réaction à l'accord pour la location d'agro-combinats par des privés », 2017,

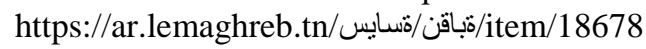

${ }^{35} \mathrm{PV}$ de l'accord entre l'UGTT et le Ministère de l'Agriculture en date du 19 juin 2017.

${ }^{36}$ Entretien avec un responsable du bureau de restructuration des terres domaniales.
} 